\title{
ON THE PROBABILITY OF AN INHERENT RELATION OF THE ELECTRICAL RESISTANCE AND THE HARDNESS OF STEEL.
}

By Carl Barus.

1. Introduction. - Some time ago ${ }^{1}$ Professor Strouhal and the writer brought forward a relation between the thermo-electric power and the specific resistance of steel varying with temper from hard to soft. More than 90 states of temper were examined, the rods being of the same kind of Stubb's steel throughout, tempered, etc., with scrupulous care. This relation, as shown in the annexed figure ( $a$ thermoelectric power in micro-volts relatively to pure silver, $s$ specific resistance in microhms per c.c., at $0^{\circ}$ C.), was remarkable in the first place for the enormous interval between the extreme values of the variables (change of electrical resistance being over three fold from soft to hard, for instance) and in the second place, from the fact that the coördinates in ques-

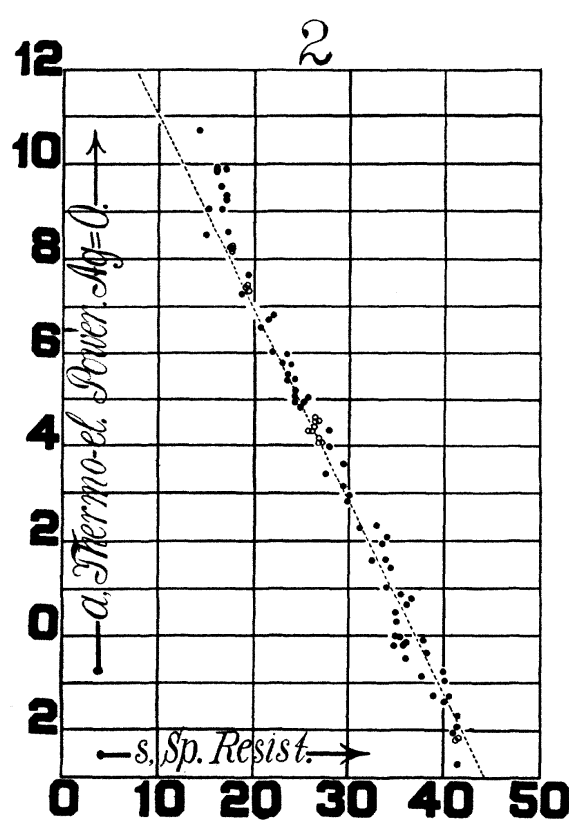

Fig. 1. tion determine a straight line. This is not true $(l . c$.$) for other alloys.$ Moreover for the case of such large variations as those in question for steel, one can hardly speak of an initial tangent.

1 Wied. Annalen, VII., 1879, p. 383 ; XI., 1880, p. 930 . Also Bulletin No. 14, U. S. Geological Survey, 1885 , chapter 2. 
The constants of the line for steel were found to be, if

$$
\begin{gathered}
a=m+n s, \\
m=\mathrm{I} 5.176, \quad n=-.4 \mathrm{I} 23,
\end{gathered}
$$

all data being expressed in c.g.s. units and referred to pure silver and $\circ^{\circ} \mathrm{C}$. Hence $m-a$, which we called thermo-electric hardness, is proportional to $s$ and is virtually referred to a standard steel rod whose specific resistance is zero. This eliminates the silver.

2. Case of Two Phases. - The two functions, $a$ and $s$, of the temper of steel between the glass hard and soft states, however sensitive for discrimination, have thus far been regarded as a mere index to that quality. It does not seem improbable that they are more than this, if we regard tempered steel as a mixture of hard and soft molecules, sufficiently intimate to give but little variation of the elastic constants, while the metal passes from hard to soft. I have already made such a supposition in an earlier paper $^{1}$ with advantage.

In such a case, let $q$ be the number of hard molecules and I $-q$ the number of soft molecules per sq. $\mathrm{cm}$. of any interface within the metal. The passage from hard to soft is thus a change of $q$ from I to $O$. Hence for conduction normal to the interface we may write

and

$$
\frac{\mathrm{I}}{s}=\frac{q}{s_{1}}+\frac{\mathrm{I}-q}{s_{2}}
$$

$$
\frac{a}{s}=q \frac{a_{1}}{s_{1}}+(\mathrm{I}-q) \frac{a_{2}}{s_{2}}
$$

where $a_{1}, s_{1}$ denote the thermo-electric power and electrical resistance of extremely hard steel, $a_{2}, s_{2}$ the same variables for extremely soft steel, while $a, s$, refer to any intermediate states of temper. Hence if equations (I) and (2) are to be compatible

$$
a=\frac{a_{2} s_{1}-a_{1} s_{2}}{s_{1}-s_{2}}+\frac{a_{1}-a_{2}}{s_{1}-s_{2}} s .
$$

In other words, the relation of $a$ and $s$ is inherently linear as it was actually found to be for steel, but not generally for alloys.

${ }^{1}$ Phys. Rev., Vol. XXIX., I909, pp. 516-524. The substanc e of this and of the above paper were given at Clark University, September 8. 
3. Case of More than Two Phases. - It is clear moreover that the linear relation will in general cease to hold, if there are more than two kinds of molecules present. In case of three groups for instance

whence

$$
\begin{aligned}
& \frac{\mathrm{I}}{s}=\frac{q_{1}}{s_{1}}+\frac{q_{2}}{s_{2}}+\frac{\mathrm{I}-q_{1}-q_{2}}{s_{3}}, \\
& \frac{a}{s}=q_{1} \frac{a_{1}}{s_{1}}+q_{2} \frac{a_{2}}{s_{2}}+\left(\mathrm{I}-q_{1}-q_{2}\right) \frac{a_{3}}{s_{3}},
\end{aligned}
$$

$a=\frac{a_{2} s_{3}-a_{3} s_{2}}{s_{3}-s_{2}}+\left(\frac{a_{3}-a_{2}}{s_{3}-s_{2}}+\frac{q_{1}}{s_{1}} \frac{s_{3}\left(a_{1}-a_{2}\right)+s_{1}\left(a_{2}-a_{3}\right)+s_{2}\left(a_{3}-a_{1}\right)}{s_{3}-s_{1}}\right) s$,

which reduces to the preceding equation (3) when $q_{2}=0$. In general, however, since $q$ is essentially variable with $s$, it follows that $a$ is no longer linear with respect to $s$. This is the probable case for alloys. To use equation (6), however, some relation between the three groups of molecules present in the ratio of $q_{1}: q_{2}: \mathrm{I}-q_{1}-q_{2}$ would have to be supplied, and it would be interesting to endeavor to derive this from investigations with alloys. If there are more phases than three the case would be correspondingly involved.

4. Conclusion. - It appears therefore probable that the electrical resistance or the thermo-electric hardness of steel, is more than a mere empiric expression of its state of temper. We may write for $r$ the ratio of the number of hard to the number of soft molecules per c.c. of tempered steel

$$
r=\frac{q}{\mathrm{I}-q}=\frac{\mathrm{I} / s_{2}-\mathrm{I} / s}{\mathrm{I} / s-\mathrm{I} / s_{1}}=\frac{\lambda_{2}-\lambda}{\lambda-\lambda_{1}},
$$

i. e., the position which the electrical conduction $\lambda$ takes between the conductions $\lambda_{2}$ and $\lambda_{1}$ of extremely soft and extremely hard steel.

From this point of view, moreover, the phenomenon of temper as exhibited in the preceding paper $(l . c$.) becomes an admirable test of a law adequately broad to express dissociation in solid media, subject to time and temperature.

BROWN UNIVERSITY, Providence, R. I. 